\title{
Comparison of Changes in the Lipid Profile of Postmenopausal Women With Early Stage Breast Cancer Treated With Exemestane or Letrozole
}

\author{
Lauren Nicole Bell, PhD, Anne Thi Phuong Nguyen, BSc, CCRP, Lang Li, PhD, \\ Zeruesenay Desta, PhD, N. Lynn Henry, MD, PhD, Daniel F. Hayes, MD, \\ Antonio C. Wolff, MD, Vered Stearns, MD, Anna Maria Storniolo, MD, and \\ David A. Flockhart, MD, PhD, for The Consortium on Breast Cancer \\ Pharmacogenomics (COBRA)
}

\begin{abstract}
Effects of aromatase inhibitor (AI) therapy on the plasma lipid profile are not clear. Here the authors describe changes in fasting lipids (total cholesterol, high-density lipoprotein [HDL], low-density lipoprotein [LDL], and triglycerides) before and after 3 months of exemestane or letrozole treatment. HDL was reduced in the entire cohort $(\mathrm{P}<.001)$ and in the exemestane group $(\mathrm{P}<.001)$ but unchanged in the letrozole group $(\mathrm{P}=.169)$. LDL was increased in the entire cohort $(\mathrm{P}=.005)$ and in the letrozole group $(\mathrm{P}=.002)$ but unchanged in the exemestane group $(\mathrm{P}=.361)$. This effect was at least partially attributable to washout of tamoxifen as only patients with prior use of tamoxifen experienced a significant increase in LDL. Baseline HDL was an independent predictor of the
\end{abstract}

change in HDL $\left(r^{2}=-0.128, \mathrm{P}<.001\right)$, and prior tamoxifen use was associated with greater increases in $L D L\left(r^{2}=0.057\right.$, $\mathrm{P}<.001)$. Use of lipid-altering medications did not protect against the exemestane-induced drop in HDL or the increase in $L D L$ observed in women with prior use of tamoxifen taking letrozole. In conclusion, AI treatment and/or washout of tamoxifen induced detrimental changes in the lipid profile of postmenopausal women with breast cancer.

Keywords: exemestane; letrozole; breast cancer; lipid profile; cholesterol

Journal of Clinical Pharmacology, 2012;52:1852-1860 (C) 2012 The Author(s)
$\mathbf{T}$ hird-generation aromatase inhibitors (AIs) are increasingly being used as endocrine therapy for postmenopausal women diagnosed with hormone

From Department of Medicine, Indiana University, Indianapolis, Indiana (Dr Bell, Ms Nguyen, Dr Li, Dr Desta, Dr Storniolo, Dr Flockhart); Department of Internal Medicine and Breast Oncology Program, Comprehensive Cancer Center, University of Michigan Health and Hospitals System, Ann Arbor, Michigan (Dr Henry, Dr Hayes); and Department of Oncology, Sidney Kimmel Comprehensive Cancer Center, Johns Hopkins University, Baltimore, Maryland (Dr Wolff, Dr Stearns). Supplementary data for this article are available at http://icp.sagepub. com/supplemental/. Submitted for publication March 2, 2011 ; revised version accepted August 28, 2011. Address for correspondence: David A. Flockhart, MD, PhD, Division of Clinical Pharmacology, Wishard Memorial Hospital, WD Myers Bldg, W7123, 1001 West 10th St, Indianapolis, IN 46202; e-mail:dflockha@iupui.edu.

DOI: $10.1177 / 0091270011424153$ receptor-positive breast cancer. Although the 2 nonsteroidal AIs (anastrozole and letrozole) and the steroidal inactivator (exemestane) all substantially decrease whole-body aromatization in postmenopausal women, ${ }^{1-4}$ the chemical structures and mechanisms of action of these compounds vary.

Patients treated with AIs experience approximately $98 \%$ inhibition of aromatase enzyme action, ${ }^{1,2,5}$ leading to a high level of estrogen deprivation. Although estrogen reduction is central to the treatment of hormone receptor-positive breast cancer, estrogens have other physiological effects. Importantly, estrogen deprivation in postmenopausal women has been shown to affect lipid concentrations, bone metabolism, and the incidence of cardiovascular disease. ${ }^{6}$ As postmenopausal women are already at increased risk for altered lipid profiles 
and cardiovascular disease, it is necessary to prospectively examine the effects of using steroidal and nonsteroidal AIs on lipid parameters in this population.

Overall, indirect comparisons of the efficacy and safety profiles of the steroidal and nonsteroidal AIs, when used in the adjuvant setting, suggest they are similar. ${ }^{4,7-9}$ Previous studies investigating the effects of exemestane and letrozole on several lipid parameters have produced mixed results. ${ }^{10-17}$ The causes of this variability in response are unknown but may be due to differences in study design and genetic and/or population differences among the patient cohorts. The COnsortium on BReast cancer phArmacogenomics (COBRA) is conducting a multicenter, prospective randomized clinical trial with a primary goal of investigating the pharmacogenomics of 2 AIs, exemestane and letrozole (ELPh trial; Exemestane and Letrozole Pharmacogenomics). A key objective of this trial is to evaluate, prospectively, the effects of 3 months of AI treatment on the fasting lipid panel.

\section{METHODS}

Participants. This prospective lipid panel analysis was conducted as one component of a prospective, multi-institutional, randomized observational openlabel clinical trial of postmenopausal women for whom treatment with an AI was appropriate. The study design is listed on http://www.clinicaltrials. gov (NCT00228956) and was previously described in detail. ${ }^{18}$ Overall, 503 women enrolled in the study from August 2005 to July 2009. Participants were eligible if they were postmenopausal, diagnosed with hormone receptor-positive ductal carcinoma in situ (DCIS/stage 0) or stage I to III invasive breast cancer, and considering AI therapy either as initial adjuvant endocrine therapy or after 1 to 5 years of tamoxifen treatment. Patients were excluded from the study if they had a history of bilateral mastectomy or radiation to the contralateral breast, prior use of an aromatase inhibitor, or a history of ovarian cancer, endometrial cancer, cancer of the fallopian tube, or primary peritoneal carcinomatosis. Patients were recruited to the cohort from the breast cancer clinics at the University of Michigan Comprehensive Cancer Center, the Sidney Kimmel Comprehensive Cancer Center at Johns Hopkins, and the Melvin and Bren Simon Cancer Center at the Indiana University School of Medicine. The institutional review board at each study site approved the study, and all participants gave written informed consent.
Patients were stratified based on prior chemotherapy, prior tamoxifen, and prior bisphosphonate use and must have completed recommended local therapy as well as any adjuvant chemotherapy, including tamoxifen, prior to initiation of aromatase inhibitor treatment. Patients were randomly assigned to receive either exemestane (Aromasin; Pfizer, Inc, New York, New York; 25 mg orally per day) or letrozole (Femara; Novartis Pharmaceuticals Corporation, East Hanover, New Jersey; 2.5 mg orally per day) for 2 years. Pretreatment medical histories, medication lists, physical examinations, and laboratory samples were obtained for each patient at baseline and after 3 months of AI treatment.

Patients were excluded from this lipid analysis if samples were not available at both the baseline and 3-month time points, if they were not fasting at the time of blood draw, or if they discontinued the assigned AI medication before the 3-month time point. Women with an established lipid-altering treatment regimen (including a statin, fibrate, and/or ezetimibe) prior to enrollment in the ELPh trial who continued on this therapy throughout the 3-month study period without any changes in type or dose of drug were included in a separate substudy. Women who started or stopped taking a lipid-altering medication or changed drug or dose during the 3-month study period were excluded from both analyses.

Sample collection and measurement of lipid profiles. Venous blood samples for lipid panel analyses were collected at the baseline and 3-month follow-up visit after an overnight (at least 12-hour) fast. Total cholesterol, high-density lipoprotein (HDL) cholesterol, low-density lipoprotein (LDL) cholesterol, and triglycerides (TG) were analyzed at the clinical laboratories at the University of Michigan Health System, the Johns Hopkins Medical Laboratories, and the Indiana University Clarian Pathology Laboratory. All test centers met Clinical Laboratory Improvement Amendments (CLIA) standards.

Statistical analysis. Basic descriptive statistics, including means, standard deviations (SD), ranges, and percentages, were used to characterize the study participants. Paired and unpaired Student $t$ tests were used to compare differences in lipid panel variables at baseline and after 3 months of AI treatment. Pearson's correlation coefficients were used to test for associations between lipid profile parameters and descriptive variables. When appropriate, stepwise regression analysis was used to take into account the linear effect of several independent variables predicting the 


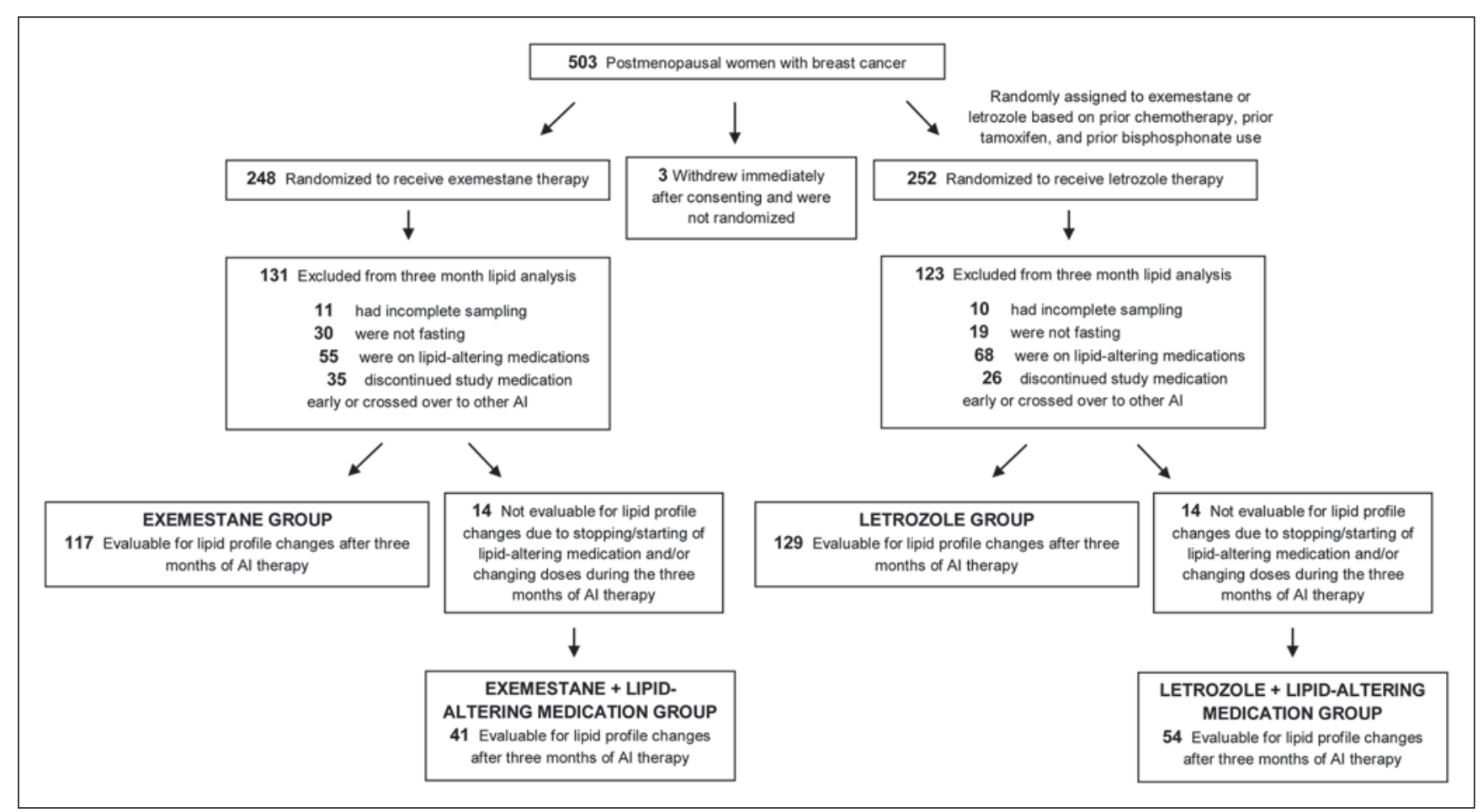

Figure 1. CONSORT diagram of included and excluded participants in this lipid analysis. AI, aromatase inhibitor.

dependent variable. Statistical analyses were performed using SPSS 16.0 for Windows (SPSS, Inc, an IBM Company, Chicago, Illinois). A $P<.05$ was considered statistically significant.

\section{RESULTS}

Study population. Of the 503 women enrolled in the ELPh clinical trial, 246 were eligible for inclusion in the overall lipid analysis (CONSORT diagram shown in Figure 1 and baseline patient characteristics shown in Table I). Women were excluded due to incomplete sampling $(\mathrm{n}=21)$, sampling under nonfasting conditions $(\mathrm{n}=49)$, use of lipid-altering medications $(n=123)$, or early discontinuation of AI treatment or crossover of AI assignment due to intolerability $(n=61)$. Patient characteristics for the individual exemestane $(n=117)$ and letrozole $(n=129)$ groups are also included in Table I.

Lipid profiles. Fasting lipid concentrations before and after AI treatment are shown in Table II. In the overall lipid analysis cohort, there was no significant change in total cholesterol $(0.3 \pm 27 \mathrm{mg} / \mathrm{dL} ; P=.847)$, but AI therapy induced a $4 \pm 9-\mathrm{mg} / \mathrm{dL}$ reduction in HDL cholesterol $(P<.001)$ and a $5 \pm 26-\mathrm{mg} / \mathrm{dL}$ increase in LDL concentration $(P=.005)$. These changes resulted in a significant increase in the LDL/HDL ratio in the entire patient cohort $(P<.001)$.

Lipid changes were also analyzed by specific agent subgroup. In the exemestane group, total cholesterol was reduced by $8 \pm 28 \mathrm{mg} / \mathrm{dL}(P=.003)$, HDL concentrations fell $(-8 \pm 9 \mathrm{mg} / \mathrm{dL} ; P<.001)$, LDL did not change significantly $(2 \pm 27 \mathrm{mg} / \mathrm{dL} ; P=.361)$, but the LDL/HDL ratio was significantly increased $(P<.001)$. In addition, triglycerides were significantly decreased by $12 \pm 42 \mathrm{mg} / \mathrm{dL}(P=.003)$. In the letrozole group, total cholesterol was increased by $8 \pm 24 \mathrm{mg} / \mathrm{dL}(P<.001)$, HDL concentrations did not change significantly $(-1 \pm 9 \mathrm{mg} / \mathrm{dL} ; P=.169)$, LDL concentrations increased $7 \pm 25 \mathrm{mg} / \mathrm{dL}(P=.002)$, and the LDL/HDL ratio was significantly increased $(P<.001)$. Overall, our data revealed a shift in HDL cholesterol distribution toward lower concentrations in the exemestane group (Figure 2, top panel) but no change in HDL in the letrozole group (Figure 2, bottom panel) after 3 months of AI therapy. This frequency distribution analysis revealed significant interindividual variation among patients.

Relationship between AI-induced changes in lipid parameters and patient characteristics. Correlates of 
Table I Baseline Characteristics of Patients Included in Lipid Panel Study

\begin{tabular}{|c|c|c|c|c|c|c|}
\hline & \multicolumn{3}{|c|}{ Overall Lipid Analysis } & \multicolumn{3}{|c|}{ Lipid-Altering Medication Analysis } \\
\hline & $\begin{array}{l}\text { Entire Cohort } \\
\quad(n=246)\end{array}$ & $\begin{array}{c}\text { Exemestane } \\
\text { Group } \\
(\mathbf{n}=117)\end{array}$ & $\begin{array}{l}\text { Letrozole } \\
\text { Group } \\
(\mathbf{n}=129)\end{array}$ & $\begin{array}{l}\text { Lipid-Altering } \\
\text { Medication } \\
\text { Cohort }(n=95)\end{array}$ & $\begin{array}{l}\text { Exemestane + } \\
\text { Lipid-Altering } \\
\text { Medication } \\
\text { Group }(\mathbf{n}=\mathbf{4 1})\end{array}$ & $\begin{array}{c}\text { Letrozole + Lipid- } \\
\text { Altering } \\
\text { Medication } \\
\text { Group }(n=54)\end{array}$ \\
\hline Age, y, mean (range) & $58(38-85)$ & $59(44-85)$ & $57(38-80)$ & $62(44-84)$ & $61(44-83)$ & $63(49-84)$ \\
\hline $\begin{array}{l}\text { Body mass index, } \mathrm{kg} / \mathrm{m}^{2} \text {, } \\
\text { mean (SD) }\end{array}$ & $29.3(6.1)$ & $29.6(6.3)$ & $29.0(5.9)$ & $31.5(6.9)$ & $30.7(5.7)$ & $32.2(7.6)$ \\
\hline \multicolumn{7}{|l|}{ Race/ethnicity, No. (\%) } \\
\hline African American & $25(10)$ & $9(8)$ & $16(12)$ & $9(9)$ & $3(7)$ & $6(11)$ \\
\hline White & $214(87)$ & $104(89)$ & $110(85)$ & $84(88)$ & $38(93)$ & $46(85)$ \\
\hline Asian & $6(2)$ & $3(3)$ & $3(2)$ & $2(2)$ & 0 & $2(4)$ \\
\hline Other & $1(<1)$ & $1(<1)$ & 0 & 0 & 0 & 0 \\
\hline $\begin{array}{l}\text { Prior chemotherapy, } \\
\text { No. }(\%)\end{array}$ & $120(49)$ & $51(44)$ & $69(53)$ & $31(33)$ & $13(32)$ & $18(33)$ \\
\hline $\begin{array}{l}\text { Prior tamoxifen use, } \\
\text { No. }(\%)\end{array}$ & $93(38)$ & $38(32)$ & $55(43)$ & $30(32)$ & $13(32)$ & $17(31)$ \\
\hline $\begin{array}{l}\text { Duration of tamoxifen } \\
\text { therapy, y, mean (SD) }\end{array}$ & $2.8(1.5)$ & $3.0(1.5)$ & $2.7(1.5)$ & $2.8(1.3)$ & $3.3(1.5)$ & $2.5(1.1)$ \\
\hline \multirow{5}{*}{$\begin{array}{l}\text { Concomitant } \\
\text { bisphosphonate use, } \\
\text { No. }(\%)\end{array}$} & 38 (15) & $19(16)$ & 19 (15) & $16(17)$ & $9(22)$ & 7 (13) \\
\hline & & \multicolumn{2}{|c|}{$\begin{array}{c}\text { Lipid-Altering Medications, } \\
\text { No. }(\%)^{\mathrm{a}}\end{array}$} & & & \\
\hline & & Statins & & $86(91)$ & 37 (90) & $49(91)$ \\
\hline & & Fibrates & & $6(6)$ & $1(2)$ & $5(9)$ \\
\hline & & Ezetimibe & & $10(11)$ & $5(12)$ & $5(9)$ \\
\hline
\end{tabular}

a. Please note that 7 women were taking 2 lipid-altering medications: statin + ezetimibe $(n=5)$ and statin + fibrate $(n=2)$.

Table II Change in Lipid Parameters After 3 Months of Aromatase Inhibitor

Therapy in the Overall Lipid Analysis Cohort $(\mathrm{n}=246)$

\begin{tabular}{|c|c|c|c|c|c|c|c|c|c|c|c|c|}
\hline & \multicolumn{4}{|c|}{ Entire Cohort $(n=246)$} & \multicolumn{4}{|c|}{ Exemestane Group $(n=117)$} & \multicolumn{4}{|c|}{ Letrozole Group $(n=129)$} \\
\hline & Baseline & 3 Months & $\begin{array}{c}\text { Change, Mean } \\
\pm \text { SD (\%) }\end{array}$ & $\begin{array}{c}P \\
\text { Value }\end{array}$ & Baseline & 3 Months & $\begin{array}{c}\text { Change, Mean } \pm \\
\text { SD (\%) }\end{array}$ & $\begin{array}{c}P \\
\text { Value }\end{array}$ & Baseline & 3 Months & $\begin{array}{c}\text { Change, Mean } \\
\pm \text { SD (\%) }\end{array}$ & $\begin{array}{c}P \\
\text { Value }\end{array}$ \\
\hline $\begin{array}{l}\text { Total } \\
\text { cholesterol, } \\
\mathrm{mg} / \mathrm{dL}\end{array}$ & $208 \pm 35$ & $208 \pm 33$ & $0.3 \pm 27(<1)$ & .847 & $212 \pm 36$ & $205 \pm 34$ & $-8 \pm 28(4)$ & .003 & $203 \pm 35$ & $211 \pm 33$ & $8 \pm 24(4)$ & $<.001$ \\
\hline HDL, mg/dL & $62 \pm 17$ & $57 \pm 17$ & $-4 \pm 9(7)$ & $<.001$ & $63 \pm 18$ & $55 \pm 16$ & $-8 \pm 9(12)$ & $<.001$ & $60 \pm 17$ & $59 \pm 17$ & $-1 \pm 9(2)$ & .169 \\
\hline $\mathrm{LDL}, \mathrm{mg} / \mathrm{dL}$ & $124 \pm 32$ & $129 \pm 29$ & $5 \pm 26(4)$ & .005 & $127 \pm 32$ & $129 \pm 28$ & $2 \pm 27(2)$ & .361 & $121 \pm 31$ & $128 \pm 30$ & $7 \pm 25(5)$ & .002 \\
\hline $\begin{array}{l}\text { LDL/HDL } \\
\text { Ratio }\end{array}$ & $2.2 \pm 0.9$ & $2.4 \pm 0.9$ & $0.2 \pm 0.6$ & $<.001$ & $2.2 \pm 0.9$ & $2.5 \pm 0.9$ & $0.3 \pm 0.6$ & $<.001$ & $2.2 \pm 0.9$ & $2.4 \pm 0.9$ & $0.2 \pm 0.5(8)$ & $<.001$ \\
\hline $\mathrm{TG}, \mathrm{mg} / \mathrm{dL}$ & $111 \pm 53$ & $106 \pm 48$ & $-5 \pm 43(5)$ & .053 & $112 \pm 53$ & $101 \pm 45$ & $-12 \pm 42(10)$ & .003 & $110 \pm 54$ & $110 \pm 50$ & $0.4 \pm 43(<1)$ & .922 \\
\hline
\end{tabular}

Values are shown as mean \pm SD. HDL, high-density lipoprotein; LDL, low-density lipoprotein; TG, triglycerides.

the changes in HDL and LDL cholesterol induced by AI therapy in the entire lipid analysis cohort $(n=246)$ summarized in Table III. Univariate analysis revealed a significant association between the AI-induced change in HDL cholesterol and baseline lipid profiles. HDL was more likely to decline after 


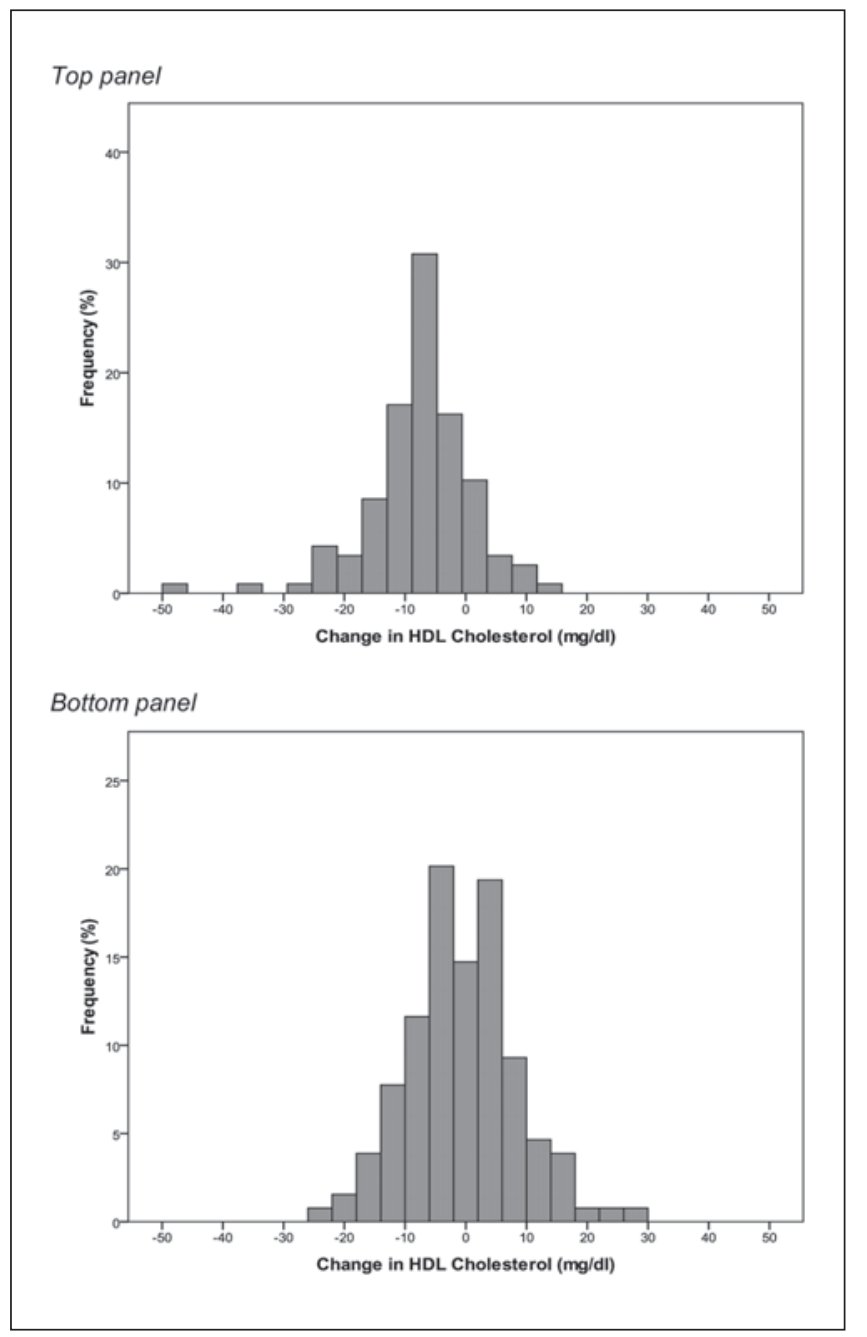

Figure 2. Distributions of the change in high-density lipoprotein (HDL) cholesterol in the exemestane group ( $n=117$; top panel) and letrozole group ( $n=129$; bottom panel) after 3 months of aromatase inhibitor (AI) therapy. The frequency ( $\%$ of patients) is shown on the $y$-axis, and the change in HDL cholesterol concentration $(\mathrm{mg} / \mathrm{dL})$ is shown on the $\mathrm{x}$-axis. The HDL categories were selected arbitrarily, and no test statistics were conducted.

3 months of therapy with either $\mathrm{AI}$ in women whose baseline total cholesterol or HDL cholesterol was high (correlation between change in HDL cholesterol with baseline total cholesterol: $r^{2}=-0.023, P=.017$ and baseline HDL cholesterol: $\left.r^{2}=-0.125, P<.001\right)$. Therefore, there was a statistically significant positive association between the AI-induced reduction in HDL cholesterol and the baseline LDL/HDL ratio $\left(r^{2}=0.054, P<.001\right)$. Upon multivariate regression analysis in which baseline total cholesterol, baseline
Table III Univariate and Multivariate Analysis of Predictors of the Change in HDL and LDL

Cholesterol After 3 Months of Aromatase

Inhibitor Therapy in the Overall Lipid Analysis Cohort $(\mathrm{n}=246)$

\begin{tabular}{|c|c|}
\hline \multicolumn{2}{|c|}{ Change Correlates } \\
\hline HDL Cholesterol & LDL Cholesterol \\
\hline $\begin{array}{c}\text { Baseline total cholesterol } \\
\left(r^{2}=-0.023, P=.017\right)\end{array}$ & $\begin{array}{c}\text { Baseline total cholesterol } \\
\left(r^{2}=-0.178, P<.001\right)\end{array}$ \\
\hline $\begin{array}{l}\text { Baseline HDL cholesterol } \\
\\
\left(r^{2}=-0.125, P<.001\right)\end{array}$ & $\begin{array}{c}\text { Baseline LDL cholesterol }^{\mathrm{a}} \\
\left(r^{2}=-0.252, P<.001\right)\end{array}$ \\
\hline $\begin{array}{l}\text { Baseline LDL/HDL ratio } \\
\left(r^{2}=0.054, P<.001\right)\end{array}$ & $\begin{array}{l}\text { Baseline LDL/HDL ratio } \\
\left(r^{2}=-0.088, P<.001\right) \\
\text { Prior tamoxifen use } \\
\left(r^{2}=0.123, P<.001\right)\end{array}$ \\
\hline
\end{tabular}

HDL, high-density lipoprotein; LDL, low-density lipoprotein.

a. Independent predictors upon stepwise regression analysis.

HDL cholesterol, and the baseline LDL/HDL ratio were considered, only baseline HDL cholesterol $\left(r^{2}=-0.125, P<.001\right)$ remained a significant predictor of the reduction in HDL following AI treatment (Figure 3, top panel). Baseline HDL cholesterol was also identified as an independent predictor of the change in HDL when the exemestane group was examined separately $\left(r^{2}=-0.253, P<.001\right)$.

The change in LDL cholesterol in the entire lipid analysis cohort was significantly correlated with baseline total cholesterol $\left(r^{2}=-0.178, P<.001\right)$, baseline LDL cholesterol $\left(r^{2}=-0.252, P<.001\right)$, and baseline LDL/HDL ratio $\left(r^{2}=-0.088, P<.001\right)$, as well as positively associated with prior tamoxifen use $\left(r^{2}=0.123, P<.001\right)$. Upon multivariate regression analysis in which baseline total cholesterol, baseline LDL cholesterol, baseline LDL/HDL ratio, and prior tamoxifen use were considered, both baseline LDL cholesterol $\left(r^{2}=-0.192, P<.001\right)$ and prior tamoxifen use $\left(r^{2}=0.051, P<.001\right)$ remained statistically significant predictors of the change in LDL cholesterol (Figure 3, bottom panel). Baseline LDL cholesterol $\left(r^{2}=0.153, P<.001\right)$ and prior use of tamoxifen $\left(r^{2}=0.098, P<.001\right)$ were also identified as independent predictors of the change in LDL cholesterol when the letrozole group was examined separately.

Prior use of tamoxifen was identified as an independent predictor of the change in LDL cholesterol; therefore, patients were stratified by this variable, and changes in lipid parameters were analyzed as shown in Supplementary Table SI. Briefly, prior use 


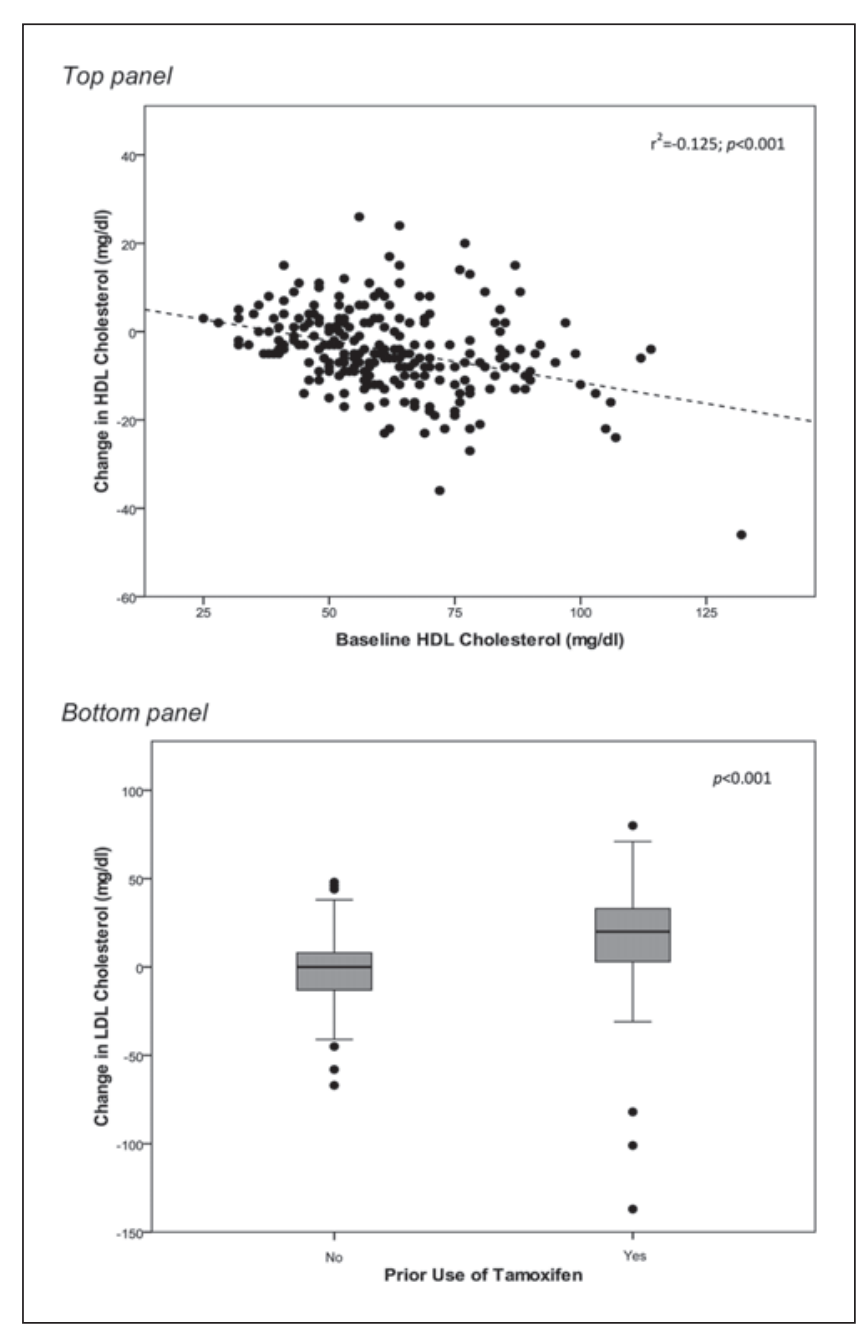

Figure 3. Significant predictors of the change in high-density lipoprotein (HDL) cholesterol (top panel) and low-density lipoprotein ( $L D L$ ) cholesterol (bottom panel) induced by aromatase inhibitor (AI) therapy in the overall lipid analysis cohort ( $n=246)$. Baseline HDL cholesterol was also identified as an independent predictor of the change in HDL when the exemestane group was examined separately $\left(r^{2}=-0.253\right.$, $P<.001)$. Prior use of tamoxifen was an independent predictor of the change in LDL cholesterol in both the overall lipid analysis cohort (bottom panel) and the letrozole group alone $(P<.001)$.

of tamoxifen did not affect the exemestane-induced reduction in HDL cholesterol $(P<.001)$ but was at least partially responsible for observed elevations in LDL cholesterol in the letrozole group, as only women who stopped tamoxifen treatment experienced a significant increase in LDL $(P<.001)$.

Lipid-altering medication cohort substudy. Of the 123 women taking a lipid-altering medication prior to initiating AI therapy, 95 were eligible for inclusion in the lipid analysis (Figure 1). In addition to the exclusion criteria described above for the overall lipid analysis, women were specifically excluded from this cohort if they did not have an established lipid-lowering treatment regimen prior to enrollment or if they changed lipid-altering drugs or doses during the 3 -month study period $(n=28)$. Baseline characteristics of patients included in the lipidaltering medication substudy are shown in Table I. Women included in this cohort were significantly older (mean \pm SD age $62.4 \pm 8.9$ years vs $58.2 \pm 7.9$ years; $P<.001$ ) and heavier (body mass index [BMI] $31.5 \pm 6.9 \mathrm{~kg} / \mathrm{m}^{2}$ vs $29.3 \pm 6.1 \mathrm{~kg} / \mathrm{m}^{2} ; P=.003$ ), but all other patient demographics and treatment histories were similar to those in the overall lipid analysis cohort (Table I).

Lipid panel measures for women included in the lipid-altering medication cohort, before and after 3 months of AI treatment, are shown in Table IV. Baseline total cholesterol, HDL, and LDL concentrations (all $P<.001)$ and LDL/HDL ratio $(P=.005)$ were significantly lower in women taking lipidaltering medications compared to those in the overall lipid analysis cohort, but triglycerides did not differ substantially between the 2 patient groups $(P=.067)$. Similar to the observation in the overall lipid analysis, 3 months of AI therapy induced a significant decrease in total cholesterol $(-7 \pm 20 \mathrm{mg} /$ $\mathrm{dL} ; P=.001$ ) and a reduction in HDL cholesterol $(-4 \pm 7 \mathrm{mg} / \mathrm{dL} ; P<.001)$. As was seen in the exemestanetreated cohort in the overall lipid analysis, women taking exemestane in the lipid-altering medication cohort also had significantly reduced total cholesterol $(-12 \pm 22 \mathrm{mg} / \mathrm{dL} ; P=.001)$, HDL cholesterol $(-5 \pm 8 \mathrm{mg} / \mathrm{dL} ; P<.001)$, and triglycerides $(-20 \pm 54$ $\mathrm{mg} / \mathrm{dL} ; P=.024)$. The only AI-induced change observed in the letrozole-treated lipid-altering medication group was a reduction in HDL cholesterol of $4 \pm 7 \mathrm{mg} / \mathrm{dL}(P=.001)$. Due to the effects of tamoxifen washout, women included in the lipid-altering medication cohort were also stratified by prior use of tamoxifen, and changes in lipid panel parameters were analyzed (Supplementary Table SII).

\section{DISCUSSION}

Results from the lipid panel study within the prospective clinical trial described here demonstrate important negative effects of 3 months of AI treatment and/or washout of tamoxifen on the fasting lipid profile of postmenopausal women with breast cancer. Adverse effects on the lipid panel are not desirable as cholesterol and triglyceride concentrations are often used as a surrogate indicator for long-term cardiovascular risk. Overall, women on exemestane 
Table IV Change in Lipid Parameters After 3 Months of Aromatase Inhibitor Therapy in the Lipid-Altering Medication Analysis ( $=95)$

\begin{tabular}{|c|c|c|c|c|c|c|c|c|c|c|c|c|}
\hline & \multicolumn{4}{|c|}{ Lipid-Altering Medication Cohort $(\mathbf{n}=\mathbf{9 5})$} & \multicolumn{4}{|c|}{$\begin{array}{c}\text { Exemestane + Lipid-Altering Medication } \\
\text { Group }(n=41)\end{array}$} & \multicolumn{4}{|c|}{$\begin{array}{l}\text { Letrozole }+ \text { Lipid-Altering Medication } \\
\text { Group }(n=54)\end{array}$} \\
\hline & Baseline & 3 Months & $\begin{array}{l}\text { Change, Mean } \\
\quad \pm \text { SD (\%) }\end{array}$ & $\begin{array}{c}P \\
\text { Value }\end{array}$ & Baseline & 3 Months & $\begin{array}{l}\text { Change, Mean } \\
\quad \pm \text { SD (\%) }\end{array}$ & $\begin{array}{c}P \\
\text { Value }\end{array}$ & Baseline & 3 Months & $\begin{array}{l}\text { Change, } \\
\text { Mean } \pm \text { SD } \\
(\%)\end{array}$ & $\begin{array}{c}P \\
\text { Value }\end{array}$ \\
\hline $\begin{array}{l}\text { Total } \\
\text { cholesterol, } \\
\mathrm{mg} / \mathrm{dL}\end{array}$ & $176 \pm 32$ & $168 \pm 30$ & $-7 \pm 20(4)$ & .001 & $180 \pm 31$ & $167 \pm 30$ & $-12 \pm 22(7)$ & .001 & $173 \pm 32$ & $169 \pm 29$ & $-3 \pm 1$ & .172 \\
\hline $\mathrm{HDL}, \mathrm{mg} / \mathrm{dL}$ & $54 \pm 15$ & $50 \pm 13$ & $-4 \pm 7(8)$ & $<.001$ & $54 \pm 12$ & $49 \pm 12$ & $-5 \pm 8(10)$ & $<.001$ & 54 & 5 & $-4 \pm 7(7)$ & .001 \\
\hline$/ \mathrm{dL}$ & $97 \pm 26$ & $95 \pm 2$ & $-3 \pm 19(3)$ & .186 & $100 \pm 26$ & $96 \pm 24$ & $-4 \pm 21(4)$ & .239 & $95 \pm 27$ & $93=$ & $-2 \pm 18(2)$ & .502 \\
\hline $\begin{array}{l}\text { LDL/HDL } \\
\text { Ratio }\end{array}$ & $1.9 \pm 0.7$ & $2.0 \pm 0.7$ & $0.1 \pm 0.5(5)$ & .071 & $1.9 \pm 0.7$ & $2.1 \pm 0.6$ & $0.2 \pm 0.5(10)$ & .123 & $1.9 \pm 0.8$ & $2.0 \pm 0.7$ & $0.1 \pm 0.5(5)$ & .310 \\
\hline $\mathrm{TG}, \mathrm{mg} / \mathrm{dL}$ & $124 \pm 59$ & $120 \pm 53$ & $-4 \pm 48(3)$ & .463 & $129 \pm 64$ & $109 \pm 43$ & $-20 \pm 54(15)$ & .024 & $120 \pm 55$ & $128 \pm 59$ & $9 \pm 40(7)$ & .112 \\
\hline
\end{tabular}

Values are shown as mean \pm SD. HDL, high-density lipoprotein; LDL, low-density lipoprotein; TG, triglycerides.

therapy exhibited reduced total and HDL cholesterol and triglycerides, whereas treatment with letrozole increased total and LDL cholesterol in women with prior use of tamoxifen. This resulted in a significant $12 \%$ and $8 \%$ elevation in the LDL/HDL ratio in the exemestane group $(P<.001)$ and the letrozole group $(P<.001)$, respectively. Women taking lipid-altering medications were not protected from changes in their lipid profile, as both the drop in HDL cholesterol induced by exemestane treatment and the elevation in LDL cholesterol in women with prior use of tamoxifen taking letrozole were observed in this cohort. Furthermore, letrozole treatment did not substantially affect HDL cholesterol in the overall analysis, but women taking both letrozole and lipid-altering medications experienced a significant reduction in HDL cholesterol.

To date, several clinical trials have proven the efficacy of the third-generation AIs such as exemestane and letrozole in the treatment of breast cancer and shown that AI treatment, in some settings, may be superior to the previously established tamoxifen treatment regimen., ${ }^{3,-9,19}$ The clinical trial described herein is the first designed as a direct, head-to-head comparison of a steroidal AI (exemestane) with a nonsteroidal AI (letrozole) in postmenopausal women with breast cancer. To the best of our knowledge, this study is also the first to independently analyze a group of women taking an AI plus lipidaltering medications, as previous reports either do not address this point or list use of medications known to affect lipids as exclusion criteria. Therefore, the unfavorable lipid profile changes after just 3 months of AI therapy/tamoxifen washout observed in our large patient cohort are an important addition to data previously reported regarding the effects of exemestane and letrozole on lipid concentrations and provide important information for prescribers to be aware of. Three recent studies have demonstrated a reduction in HDL cholesterol induced by exemestane treatment, as was observed in the present study. ${ }^{10-12}$ In 2 conflicting reports, a largely neutral effect of exemestane on the lipidemic profile was demonstrated. ${ }^{13-15}$ As is the case for exemestane, clinical trials investigating lipid effects of the nonsteroidal AI letrozole also have divergent findings. In an early study, letrozole treatment was shown to significantly increase total and LDL cholesterol. ${ }^{16}$ Subsequently, Wasan et a ${ }^{17}$ demonstrated that letrozole therapy did not significantly alter lipid parameters.

Differences in study design, prior tamoxifen use, and inclusion/exclusion of patients on lipid-altering medications likely contribute to the variability seen in AI-induced changes, or lack thereof, in lipid profiles. For example, we observed that prior use of tamoxifen is a significant independent predictor of increased LDL cholesterol in both the entire patient cohort and in the letrozole cohort. There is definitive evidence that tamoxifen reduces LDL cholesterol $^{20-26}$; therefore, our observation is consistent with previous tamoxifen studies. Although women in our study had differing durations of tamoxifen therapy, a plausible explanation is that this treatment reduced their LDL cholesterol and, upon cessation of tamoxifen therapy, they experienced loss of this positive effect on LDL from tamoxifen. It is important to note that in women with prior use of tamoxifen, elevations in LDL cholesterol were not as pronounced in the exemestane group as in the letrozole group, especially in the lipid-altering medication cohort. This raises the possibility that letrozole treatment 
itself may, in fact, increase LDL cholesterol in some women.

Although exemestane and letrozole are both classified as AIs, there are substantial differences in their chemical structures and mechanisms of action. Exemestane is a steroid-based inactivator that functions as a suicidal inhibitor of aromatase enzyme. Importantly, an early in vitro study showed that a metabolite of exemestane, 17-hydroxy exemestane, may elicit androgenic effects through binding to the androgen receptor. ${ }^{27}$ Because previous work has shown that exogenous androgens have a significant detrimental effect on HDL cholesterol in postmenopausal women, ${ }^{28}$ we hypothesize that this may be a potential mechanism by which exemestane and/or 17-hydroxy exemestane could reduce HDL cholesterol. In addition, baseline total and HDL cholesterol were slightly higher in the exemestane group, and because both were identified as predictors of the change in HDL cholesterol, this cannot be excluded as a contributing factor to the reduction in HDL cholesterol observed in patients treated with exemestane. Metabolism of letrozole does not lead to production of an androgenic metabolite; therefore, less is currently known about mechanisms by which letrozole may potentially induce changes in lipids.

Regardless of how and to what extent exemestane or letrozole treatment and/or tamoxifen washout reduce HDL and increase LDL cholesterol, respectively, a detrimental effect on the lipid profile is not ideal as postmenopausal women are already at increased risk for cardiovascular disease. As the potential use of AIs expands beyond the context of adjuvant therapy for postmenopausal women diagnosed with breast cancer to chemoprevention for high-risk women, ${ }^{29}$ consideration of the risk-benefit ratio is increasingly important. The large interindividual variability of AI-induced changes in HDL cholesterol is shown in Figure 2. Future study will be directed at identifying women who may benefit most from treatment with an $\mathrm{AI}$ and/or tamoxifen while also avoiding unfavorable adverse effects.

The effects of AI-induced changes in cholesterol on the risk of cardiovascular events have not been established, although a recent review of the AI clinical trials showed no significant increases in cardiovascularrelated adverse events compared to tamoxifen or placebo. $^{30}$ In addition, a meta-analysis of randomized clinical trials with AIs showed no increase in nonbreast deaths with AI treatment. ${ }^{3}$ However, it would likely take many additional years of study to clearly define the cardiovascular effects of AI therapy. A paradox exists with tamoxifen, which has a positive effect on the lipid profile ${ }^{20-26}$ and may reduce the risk of cardiovascular disease ${ }^{31}$ but increases the incidence of thromboembolic events and ischemic stroke. ${ }^{6}$ Because AIs do not have estrogenic agonist effects, it is unlikely that a similar contradiction exists with AIs. Nonetheless, results from this lipid analysis support the notion that monitoring of cardiovascular morbidity and mortality in all long-term AI trials will be necessary. In addition, use of lipid-altering drugs did not convey protection from the AI-induced adverse effects on HDL cholesterol, which could potentially increase cardiovascular risk over time. Furthermore, women who experienced the greatest changes in lipids (that resulted in starting/ stopping or dose adjustment of lipid-altering medications) were not included in these analyses, and this important point should be taken into consideration when interpreting study results.

In conclusion, we observed an unfavorable effect of AI treatment alone or in combination with tamoxifen washout on the lipid panel of postmenopausal women with breast cancer and found that use of lipid-altering medications did not protect against the HDL-lowering effects of exemestane therapy. A better understanding of the side effect profile, along with identification of the genetic factors that can predict adverse events, may eventually provide the framework for making optimal AI therapy decisions for women with breast cancer.

Financial disclosure: Pharmacogenetics Research Network Grant Number U-01 GM61373 (DAF). LNB is supported in part by a Clinical Pharmacology Training Grant Number 5T32-GM08425 (DAF) from the National Institute of General Medical Sciences, National Institutes of Health, Bethesda, Maryland; grant numbers M01-RR000042 (UM), M01-RR00750 (IU), and M01-RR00052 (JHU) from the National Center for Research Resources (NCRR), a component of the National Institutes of Health; grants from Pfizer (DFH), Novartis Pharma AG (DFH), and Fashion Footwear Association of New York/QVC Presents Shoes on Sale (DFH). The study was also funded by Pfizer (DAF, DFH, VS, AMS), Novartis (DAF, DFH, VS), AstraZeneca (DAF, NLH, DFH), Lilly (NLH), and BiPar Sciences (NLH).

Consultant-LabCorp (DAF), Roche Molecular Diagnostics (DAF), Wyeth (VS), Pfizer (DFH), Honoraria-Pfizer (AMS), Pfizer (DFH), AstraZeneca (VS).

\section{REFERENCES}

1. Geisler J, King N, Anker G, et al. In vivo inhibition of aromatization by exemestane, a novel irreversible aromatase inhibitor, in postmenopausal breast cancer patients. Clin Cancer Res. 1998;4(9): 2089-2093

2. Geisler J, Haynes B, Anker G, Dowsett M, Lonning PE. Influence of letrozole and anastrozole on total body aromatization and plasma estrogen levels in postmenopausal breast cancer patients evaluated in a randomized, cross-over study. J Clin Oncol. 2002; 20(3):751-757. 
3. Dowsett M, Cuzick J, Ingle J, et al. Meta-analysis of breast cancer outcomes in adjuvant trials of aromatase inhibitors versus tamoxifen. J Clin Oncol. 2010;28(3):509-518.

4. Winer EP, Hudis C, Burstein HJ, et al. American Society of Clinical Oncology technology assessment on the use of aromatase inhibitors as adjuvant therapy for postmenopausal women with hormone receptor-positive breast cancer: status report 2004. J Clin Oncol. 2005;23(3):619-629.

5. Miller WR. Aromatase inhibitors: mechanism of action and role in the treatment of breast cancer. Semin Oncol. 2003;30(4) (suppl 14):3-11.

6. Visvanathan K, Chlebowski RT, Hurley P, et al. American Society of Clinical Oncology clinical practice guideline update on the use of pharmacologic interventions including tamoxifen, raloxifene, and aromatase inhibition for breast cancer risk reduction. J Clin Oncol. 2009;27(19):3235-3258.

7. Baum M, Budzar AU, Cuzick J, et al. Anastrozole alone or in combination with tamoxifen versus tamoxifen alone for adjuvant treatment of postmenopausal women with early breast cancer: first results of the ATAC randomised trial. Lancet. 2002; 359(9324):2131-2139.

8. Goss PE, Ingle JN, Martino S, et al. A randomized trial of letrozole in postmenopausal women after five years of tamoxifen therapy for early-stage breast cancer. N Engl J Med. 2003;349(19): 1793-1802.

9. Coombes RC, Hall E, Gibson LJ, et al. A randomized trial of exemestane after two to three years of tamoxifen therapy in postmenopausal women with primary breast cancer. $N$ Engl J Med. 2004;350(11):1081-1092.

10. Lonning PE, Geisler J, Krag LE, et al. Effects of exemestane administered for 2 years versus placebo on bone mineral density, bone biomarkers, and plasma lipids in patients with surgically resected early breast cancer. J Clin Oncol. 2005;23(22): 5126-5137.

11. Francini G, Petrioli R, Montagnani A, et al. Exemestane after tamoxifen as adjuvant hormonal therapy in postmenopausal women with breast cancer: effects on body composition and lipids. Br J Cancer. 2006;95(2):153-158.

12. Montagnani A, Gonnelli S, Cadirni A, et al. The effects on lipid serum levels of a 2-year adjuvant treatment with exemestane after tamoxifen in postmenopausal women with early breast cancer. Eur J Intern Med. 2008;19(8):592-597.

13. Markopoulos C, Polychronis A, Dafni U, et al. Lipid changes in breast cancer patients on exemestane treatment: final results of the TEAM Greek substudy. Ann Oncol. 2009;20(1):49-55.

14. Markopoulos C, Chrissochou M, Michailidou A, et al. Effect of exemestane on the lipidemic profile of post-menopausal operable breast cancer patients following 5-7 years of adjuvant tamoxifen: preliminary results of the ATENA substudy. Anticancer Drugs. 2005;16(8):879-883.

15. Markopoulos C, Dafni U, Misitzis J, et al. Extended adjuvant hormonal therapy with exemestane has no detrimental effect on the lipid profile of postmenopausal breast cancer patients: final results of the ATENA lipid substudy. Breast Cancer Res. 2009; 11(3):R35.
16. Elisaf MS, Bairaktari ET, Nicolaides C, et al. Effect of letrozole on the lipid profile in postmenopausal women with breast cancer. Eur J Cancer. 2001;37(12):1510-1513.

17. Wasan KM, Goss PE, Pritchard PH, et al. The influence of letrozole on serum lipid concentrations in postmenopausal women with primary breast cancer who have completed 5 years of adjuvant tamoxifen (NCIC CTG MA.17L). Ann Oncol. 2005; 16(5):707-715.

18. Henry NL, Giles JT, Ang D, et al. Prospective characterization of musculoskeletal symptoms in early stage breast cancer patients treated with aromatase inhibitors. Breast Cancer Res Treat. 2008; 111(2):365-372.

19. Iwase H. Current topics and perspectives on the use of aromatase inhibitors in the treatment of breast cancer. Breast Cancer. 2008;15(4):278-290.

20. Love RR, Wiebe DA, Feyzi JM, Newcomb PA, Chappell RJ. Effects of tamoxifen on cardiovascular risk factors in postmenopausal women after 5 years of treatment. J Natl Cancer Inst. 1994; 86(20):1534-1539.

21. Dziewulska-Bokiniec A, Wojtacki J, Skokowski J, Kortas B. The effect of tamoxifen treatment on serum cholesterol fractions in breast cancer women. Neoplasma. 1994;41(1):13-16.

22. Thangaraju M, Kumar K, Gandhirajan R, Sachdanandam P. Effect of tamoxifen on plasma lipids and lipoproteins in postmenopausal women with breast cancer. Cancer. 1994;73(3):659-663.

23. Grey AB, Stapleton JP, Evans MC, Reid IR. The effect of the antiestrogen tamoxifen on cardiovascular risk factors in normal postmenopausal women.JClinEndocrinolMetab.1995;80(11):3191-3195. 24. Vrbanec D, Reiner Z, Belev B, Plestina S. Changes in serum lipid and lipoprotein levels in postmenopausal patients with node-positive breast cancer treated with tamoxifen. Tumori. 1998;84(6):687-690.

25. Sharma D, Sharma U, Bhatnagar VB, Singh VS. A study of the effect of tamoxifen on serum lipoprotein profiles in premenopausal and postmenopausal women with breast carcinoma and associated risk of cardiovascular disease. Indian J Med Sci. 2001;55(7):359-365.

26. Ntukidem NI, Nguyen AT, Stearns V, et al. Estrogen receptor genotypes, menopausal status, and the lipid effects of tamoxifen. Clin Pharmacol Ther. 2008;83(5):702-710.

27. Ariazi EA, Leitao A, Oprea TI, et al. Exemestane's 17-hydroxylated metabolite exerts biological effects as an androgen. Mol Cancer Ther. 2007;6(11):2817-2827.

28. Lovejoy JC, Bray GA, Bourgeois MO, et al. Exogenous androgens influence body composition and regional body fat distribution in obese postmenopausal women: a clinical research center study. J Clin Endocrinol Metab. 1996;81(6):2198-2203.

29. Puntoni M, Decensi A. The rationale and potential of cancer chemoprevention with special emphasis on breast cancer. Eur $J$ Cancer. 2009;45(suppl 1):346-354.

30. Janni W, Hepp P. Adjuvant aromatase inhibitor therapy: outcomes and safety. Cancer Treat Rev. 2010;36(3):249-261.

31. Ewer MS, Gluck S. A woman's heart: the impact of adjuvant endocrine therapy on cardiovascular health. Cancer. 2009;115(9): 1813-1826.

For reprints and permission queries, please visit SAGE s Web site at http://www.sagepub.com/journalsPermissions.nav. 